\section{Pulsatile ocular blood flow change after treatment with systemic steroid in patients with Graves' ophthalmopathy}

C-C Tsai ${ }^{1,2,3}, \mathrm{H}-\mathrm{C} \mathrm{Kau}{ }^{2,4}, \mathrm{H}-\mathrm{H}$ Tsai ${ }^{1,2}, \mathrm{~S}-\mathrm{C} \mathrm{Kao}{ }^{1,2}$ and W-M Hsu',

\begin{abstract}
Purpose Our previous study has demonstrated an impairment of pulsatile ocular blood flow (POBF) in patients with Graves'ophthalmopathy (GO). Here we further evaluate the haemodynamic change in GO patients after systemic steroid.

Methods In a prospective, interventional, consecutive clinical case series, all patients with active and moderately severe GO who underwent systemic steroid treatment were evaluated. The change of POBF and the clinical activity and severity of the disease were assessed. Results In total, 11 patients underwent intravenous methylprednisolone pulse therapy followed by 2-month oral prednisolone therapy. POBF improved from a mean value of 476.5 to $614.7 \mu 1 / \mathrm{min}(P<0.001)$ after treatment. Likewise, the mean clinical activity score reduced from 4.8 to 1.6. However, systemic steroid had less effect on the severity of GO. It also revealed that there is a greater improvement of POBF in those with more clinical activity score $(>4)$ at the onset. Conclusions Following treatment with systemic steroid we have demonstrated a significant improvement in POBF in patients with GO.
\end{abstract}

Eye (2006) 20, 1025-1029. doi:10.1038/sj.eye.6702070; published online 26 August 2005

Keywords: Graves' ophthalmopathy; pulsatile ocular blood flow; steroid

\section{Introduction}

Graves' ophthalmopathy (GO), even in its mild expression, profoundly affects the quality of life of patients. Although most GO patients have a mild and nonprogressive clinical course that requires only supportive treatment,

lagophthalmic (exposure) keratitis and optic neuropathy can, in rare instances, lead to severe visual impairment. Therefore, an adequate assessment of GO is important both in the decision as to whether ophthalmopathy should be treated and which type of treatment could achieve a better outcome. It often relies on the assessment of two different features, the severity and the activity of the disease. ${ }^{1}$ Bartalena $e a^{1}$ had suggested the severity of ophthalmopathy can be assessed on the basis of an overall evaluation of the degree of proptosis, diplopia, and optic neuropathy. Mourits et $a l^{2}$ also proposed a clinical activity score (CAS), which ranged from 0 to 10 points based on the clinical manifestation of inflammation (pain, redness, swelling, impairment of visual acuity, and impairment of eye movement), to evaluate the activity of the ophthalmopathy. They further showed that patients with CAS $\geq 4$ had a favourable outcome of immunosuppressive treatment (oral prednisone or retrobulbar irradiation). ${ }^{3}$ There are also many studies using other parameters to assess the treatment outcome of GO, including exophthalmometer readings, ophthalmopathy index, ${ }^{4}$ total eye score, ${ }^{5}$ NOSPECS class or grade, ${ }^{6}$ and the octreotide scintigraphy. ${ }^{7}$ However, it may cause a great deal of controversy over the results depending on which parameters have been used.

By using pulsatile ocular blood flow (POBF) measurement, our previous study demonstrated a significant haemodynamic impairment in patients with GO, compared
'Department of Ophthalmology, Taipei Veterans General Hospital, Taiwan

${ }^{2}$ Department of Ophthalmology, School of Medicine, National YangMing University, Taiwan

${ }^{3}$ Institute of Clinical Medicine, National YangMing University, Taiwan

${ }^{4}$ Department of Ophthalmology, Taoyuan Veterans Hospital, Taiwan

Correspondence: S-C Kao, Department of Ophthalmology, Taipei Veterans General Hospital No. 201, Sec. 2, Shih-Pai Road, Taipei, Taiwan, ROC Tel: + 88622875 7325; Fax: + 886225551303 .

E-mail: Sckau123@

yahoo.com

Received: 16 February 2005 Accepted in revised form: 13 July 2005

Published online: 26 August 2005

No public and private supports

None of the authors have any commercial interest in the materials mentioned herein 
with Graves' patients without eye disease and normal individuals. ${ }^{8}$ Even though the mechanisms are still currently under investigation, the impaired circulation in GO patients may be explained by the mechanical effects of an increase in the volume of the retrobulbar fibroadipose tissue and extraocular muscles within the limited-space of pear-shaped orbit. Based on these findings, in this paper, we investigate whether POBF can be increased in patients with GO who have received systemic steroid treatment.

\section{Materials and methods}

Between September 2002 and June 2004, all patients who had active and moderately severe GO and underwent systemic steroid at Taipei Veterans General Hospital were prospectively eligible for study. The diagnosis of GO was based on the criteria proposed by Bartley and Gorman. ${ }^{9}$ Active GO was defined as a CAS of four or more points suggested by Mourits et al. ${ }^{3}$ In agreement with Bartalena et $a l_{,}{ }^{1}$ the severity of the disease was evaluated on the basis of the degree of proptosis, diplopia, and optic neuropathy. Proptosis was defined as 'mild': 19-20 mm; 'moderate': 21-22 mm; 'marked': > 23 mm by Hertel's exophthalmometer readings (mean normal value in our Taiwan population is $13.90 \pm 2.33 \mathrm{~mm}$ ). Diplopia was defined as 'mild': intermittent; 'moderate': inconstant; 'marked': constant. The severity of optic neuropathy was defined as follows: 'mild': subclinical visual impairment; 'moderate': visual acuity: 6/7.5-6/12; 'marked': visual acuity: <6/12.

Moderately severe ophthalmopathy was defined with at least one moderate, or two of the above mild manifestations. Study subjects were excluded if they had any ocular disease other than GO or with a history of surgical decompression, radiation therapy, pregnancy, diabetes mellitus or a severe systemic disease. A complete ophthalmic examination, including bestcorrected visual acuity, proptosis (measured by a Hertel exophthalmometer), ocular motility, constancy of diplopia (intermittent, present only when fatigued; inconstant, present in secondary positions of gaze; constant, present in primary and reading positions), optic nerve function (assessed by recording of the corrected visual acuity, funduscopy, pupil reactions, colour vision, and/or evidence of orbital apex compression on computed tomographic scanning), intraocular pressure (IOP), and slit-lamp biomicroscopy was performed in all patients. All subjects were examined by the same ophthalmologist on the day before and at 2 months after the start of treatment. General clinical conditions and blood glucose were also carefully monitored throughout the treatment. The institutional review board approved the study; informed consents were obtained from the patients.

Corticosteroid pulse therapy was performed with the following schedule. Methylprednisolone was administered intravenously at a daily dose of $1 \mathrm{~g}$ for 3 days. Oral prednisone therapy following intravenous methylprednisolone pulse therapy was performed at an initial daily dose of $0.5 \mathrm{mg} / \mathrm{kg}$ for 2 weeks, and then tapered slowly to discontinuation approximately 2 months in all patients. The activity and severity of GO and POBF were evaluated before and after treatment. On each occasion, POBF was measured three times according to the method of our previous study. ${ }^{8}$ The examiner of POBF was masked for the pretreatment assessment when performing the post-treatment assessment.

\section{Statistical analysis}

Statistical analysis was carried out using SPSS 11.0 for Windows (SPSS Inc, Chicago, IL, USA). Data are expressed as means and standard deviations (SD). Comparisons between baseline and after treatment were performed with paired Student's $t$-test. Multivariate linear regression was used to adjust the effects of IOP on POBF. Statistical significance was assumed at the $P<0.05$ level.

\section{Results}

A total of 12 patients met the inclusion criteria; however, one withdrew after developing tarry stool with the treatment of intravenous methylprednisolone. Data of 22 eyes of 11 patients (four men and seven women) with a mean age of 52.2 (SD 15.5) years (range 32-78 years) were analysed. Among the 11 patients, three patients were taking drugs for hyperthyroidism (methimazole or propylthiouracil), and all kept using the same medication during the study. None received any other concurrent medication. Mean IOP was decreased from 21.5 to $20.4 \mathrm{mmHg}(P=0.003)$. The data of clinical activity and severity of GO and POBF are shown in Table 1.

The clinical activity scores decreased significantly from 4.8 (SD 1.5) to 1.6 (SD 1.0, $P<0.001$ ) (Figure 1). Mean of exophthalmometer readings at baseline decreased slightly from $20.5 \mathrm{~mm}$ (SD 3.9) to $20.4 \mathrm{~mm}$ (SD 3.9, $P=0.056$ ). In 10 of the 11 patients, diplopia was observed before the treatment. Diplopia was improved in four patients by the therapy, and most (three patients) presented with intermittent diplopia at baseline. Improvement of best-corrected visual acuity of a variable amount was found in six $(86 \%)$ of seven patients with optic neuropathy (Table 1). Case 10 
Table 1 Change of clinical activity score (CAS), severity of Graves' ophthalmopathy (GO), and pulsatile ocular blood flow (POBF) in patients with GO after systemic steroid

\begin{tabular}{|c|c|c|c|c|c|c|c|c|c|c|c|}
\hline & & \multicolumn{2}{|c|}{ CAS } & \multicolumn{2}{|c|}{ Proptosis } & \multicolumn{2}{|c|}{ Diplopia } & \multicolumn{2}{|c|}{ Optic neuropathy } & \multicolumn{2}{|c|}{ POBF $(\mu l / m i n)$} \\
\hline & & Pre-Tx & Post-Tx & Pre-Tx & Post-Tx & Pre-Tx & Post-Tx & Pre-Tx & Post-Tx & Pre-Tx & Post-Tx \\
\hline \multirow[t]{2}{*}{1} & OD & 6 & 2 & 25 & 25 & Marked & Marked & $6 / 12$ & $6 / 10$ & 427 & 510 \\
\hline & OS & 6 & 3 & 24.5 & 24.5 & Marked & Marked & $6 / 12$ & $6 / 10$ & 420 & 590 \\
\hline \multirow[t]{2}{*}{2} & OD & 3 & 2 & 19 & 19 & Marked & Marked & No & No & 371 & 535 \\
\hline & OS & 5 & 2 & 19 & 19 & Marked & Marked & No & No & 369 & 519 \\
\hline \multirow[t]{2}{*}{3} & OD & 4 & 0 & 19 & 18.5 & Moderate & Moderate & No & No & 490 & 741 \\
\hline & OS & 5 & 1 & 20 & 20 & Moderate & Moderate & No & No & 458 & 635 \\
\hline \multirow[t]{2}{*}{4} & OD & 4 & 0 & 20 & 19.5 & Mild & No & No & No & 621 & 847 \\
\hline & OS & 2 & 0 & 22 & 21 & Mild & No & No & No & 720 & 960 \\
\hline \multirow[t]{2}{*}{5} & OD & 2 & 0 & 20.5 & 20 & Mild & No & No & No & 445 & 611 \\
\hline & OS & 4 & 1 & 19 & 19 & Mild & No & $6 / 20$ & $6 / 10$ & 408 & 545 \\
\hline \multirow[t]{2}{*}{6} & OD & 7 & 2 & 10 & 10 & Moderate & Mild & No & No & 405 & 657 \\
\hline & OS & 7 & 2 & 11 & 11 & Moderate & Mild & No & No & 483 & 690 \\
\hline \multirow[t]{2}{*}{7} & OD & 6 & 2 & 21 & 21 & Mild & No & No & No & 457 & 531 \\
\hline & OS & 6 & 2 & 24.5 & 24.5 & Mild & No & No & No & 516 & 606 \\
\hline \multirow[t]{2}{*}{8} & OD & 6 & 2 & 19 & 19 & Moderate & Moderate & No & No & 400 & 501 \\
\hline & OS & 7 & 3 & 19 & 19 & Moderate & Moderate & No & No & 415 & 554 \\
\hline \multirow[t]{2}{*}{9} & OD & 5 & 1 & 19 & 18.5 & No & No & $6 / 60$ & $6 / 7.5$ & 425 & 651 \\
\hline & OS & 5 & 2 & 20 & 20 & No & No & $3 / 60$ & $6 / 60$ & 386 & 492 \\
\hline \multirow[t]{2}{*}{10} & OD & 4 & 3 & 23 & 23 & Mild & Mild & $6 / 15$ & $6 / 15$ & 453 & 420 \\
\hline & OS & 4 & 2 & 22 & 22 & Mild & Mild & $6 / 12$ & $6 / 8.6$ & 512 & 520 \\
\hline \multirow[t]{2}{*}{11} & OD & 4 & 2 & 21 & 21 & Mild & Mild & No & No & 610 & 668 \\
\hline & OS & 4 & 2 & 22 & 22 & Mild & Mild & No & No & 692 & 740 \\
\hline
\end{tabular}

Tx: treatment.

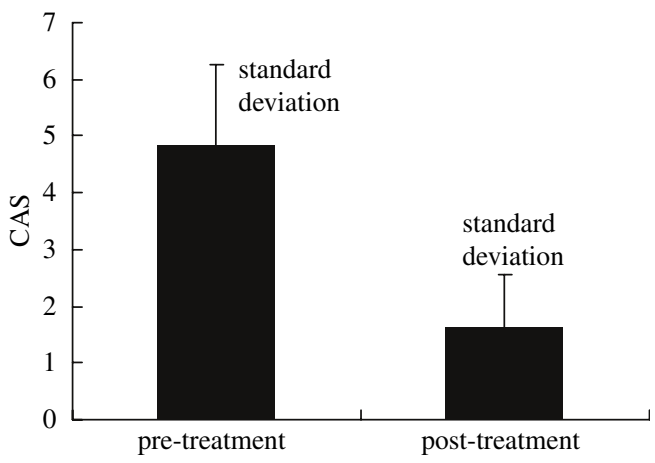

Figure 1 Change of clinical activity score (CAS) in the GO patients after systemic steroid.

was the only patient who had optic neuropathy for more than 3 months and showed poor response to systemic steroid.

Mean POBF increased by $138.2 \mu \mathrm{l} / \mathrm{min}(29 \%)$ from a baseline of $476.5 \mu \mathrm{l} / \mathrm{min}(P<0.001)$ after treatment (Figure 2). After adjusting for the factor of IOP, the increase of POBF after systemic steroid still reached statistical significance $(P<0.001)$. The change in POBF after systemic steroid treatment was further analysed according to the clinical activity score at the onset.

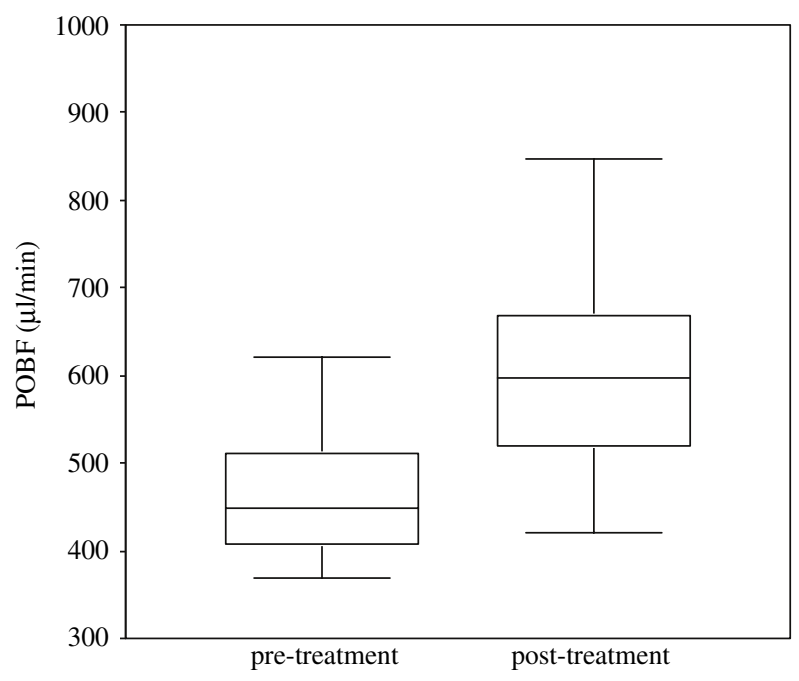

Figure 2 Boxplot showing the distribution of POBF in the GO patients before and after systemic steroid.

Subjects with CAS values of less than 4 (10 eyes) showed a decrease in POBF of $24 \%$ after treatment. Those with a CAS $>4$ (12 eyes) had a $34 \%$ decrease in their POBF after treatment (Table 2). 
Table 2 Change in pulsatile ocular blood flow (POBF) after systemic steroid treatment based on clinical activity score (CAS)

\begin{tabular}{lcc}
\hline & \multicolumn{2}{c}{$\operatorname{POBF}(\mu l / \mathrm{min})$} \\
\cline { 2 - 3 } & Pre-Tx & Post-Tx \\
\hline CAS $\leq 4$ (10 eyes) & 532 & 659 \\
CAS $>4$ (12 eyes) & 430 & 578 \\
\hline
\end{tabular}

Tx: treatment.

\section{Discussion}

The current study revealed that the POBF in GO patients improved after systemic steroid. Langham $e a^{10}$ first adapted the pneumotonometer to measure the pulsatile change of IOP and converted it into a volume pulse to obtain a measure of POBF. POBF measurement is a relatively inexpensive and noninvasive method in ocular blood flow studies, and it has been shown to be operated with acceptable reproducibility. ${ }^{11,12}$ POBF primarily reflects the choroidal circulation and provides diagnostic value especially in detecting ischemic response in ocular tissue, although it is suggested to be more useful for studying intraindividual blood flow changes (ie before and after therapy comparison) rather than interindividual comparison. ${ }^{13}$ It has been clinically found to be reduced in a variety of ocular diseases including glaucoma ${ }_{1}^{14}$ age-related macular degeneration, ${ }^{15,16}$ and diabetic retinopathy. ${ }^{17}$ Our previous study has shown that GO patients had significantly reduced POBF as compared to those with Graves' disease only or healthy subjects. ${ }^{8} \mathrm{GO}$ is an autoimmune disorder, in which orbital connective tissues often undergo a dramatic remodelling, including intense inflammation and accumulation of the hydrophilic glycosaminoglycan. This accumulation results in edematous extraocular muscles in conjunction with orbital connective and adipose tissues expansion is currently believed to underlie much of the tissue dysfunction associated with GO. Based on anatomic considerations, expansion of connective tissue or swollen muscles at the apex of the orbit and lack of forward mobility of orbital contents due to tight attachment of connective tissue to the orbital walls may result in compromised circulation within the orbit in GO patients. ${ }^{18}$ Engorgement of the superior ophthalmic vein (SOV) and decrease or reversed blood flow of SOV have also been reported in patients with GO. ${ }^{19,20}$ Somer et $a l^{21}$ further noted that the computed tomographic findings of coexistence of horizontal and vertical extraocular muscle enlargement and apical crowding of the orbit seemed to contribute to significant decreases in blood flow velocity of SOV in colour Doppler imaging study. Li et $a l^{22}$ reported that the blood flow velocity in central retinal artery were significantly lower in thyroidassociated ophthalmopathy as compared to those of normal subjects.

In our study, the improvement of POBF in most patients after therapy may be attributed to the 'medical decompression' effect of corticosteroids. In spite of other novel treatments such as somatostatin analogues, antioxidants, and development of cytokine antagonists, corticosteroids remain one of the important treatments for GO. It is particularly effective not only on soft tissue inflammation and optic neuropathy but also on extraocular muscle dysfunction (if not associated with fibrotic changes). Our study showed that clinical activity scores reduced in all patients after therapy. Further analysis revealed that there is a greater improvement of POBF in those with more clinical activity score $(>4)$ before treatment commenced, which is compatible with the result of Mourits' study. ${ }^{3}$ The improvement of extraocular muscle movements especially in those patients with mild diplopia at baseline suggests that they were mainly caused by retro- and periorbital edema rather than fibrosis. However, this treatment had a limited effect on proptosis, which is comparable with the results of previous studies. ${ }^{23}$

Improvements in vision and POBF were observed after treatment in most patients with optic neuropathy. These improvements are possibly mediated through improved ocular blood flow. Since POBF primarily reflects the choroidal circulation and the main blood supply to the optic disc and the choroid is both from the posterior ciliary arteries, the increase in choroidal circulation after therapy may be associated with improvement in optic disc circulation. One patient (case 10) did not respond well to systemic steroid in vision and POBF. It is possible that the long-standing inflammation caused irreversible fibrotic change on retro-orbital connective tissues, which caused permanent compromised ocular circulation. Therefore, it is mandatory that GO patients suffered from inadequate ocular blood flow need more aggressive monitor and treatment, because long-term ocular blood flow deficits could induce optic nerve or retinal ganglion cell damage such as those found in the patients with normal-tension glaucoma.

In conclusion, the observations we report here coupled with our earlier findings suggest that POBF

measurement is an objective and quantitative indicator of the efficacy of the treatment on patients with GO, and it is potentially important in the decision of whether a patient needs to be treated. However, further study is warranted to understand the relationship between ocular haemodynamics and progression of the GO. 


\section{Acknowledgements}

This work was presented in part as a poster at the annual meeting of American Academy of Ophthalmology, New Orleans, 2004.

\section{References}

1 Bartalena L, Pinchera A, Marcocci C. Management of Graves' ophthalmopathy: reality and perspectives. Endocr Rev 2000; 21: 168-199.

2 Mourits MP, Koornneef L, Wiersinga WM, Prummel MP, Berghout A, van der Gaag $\mathrm{R}$ et al. Clinical criteria for the assessment of disease activity in Graves' ophthalmopathy: a novel approach. Br J Ophthalmol 1989; 73: 639-644.

3 Mourits MP, Prummel MF, Wiersinga WM, Koornneef L. Clinical activity score as a guide in the management of patients with Graves' ophthalmopathy. Clin Endocrinol 1997; 47: 9-14.

4 Donaldson SS, Bagshaw MA, Kriss JP. Supervoltage orbital radiotherapy for Graves' ophthalmopathy. J Clin Endocrinol Metab 1973; 37: 276-285.

5 Prummel MF, Mourits MP, Berghout A, Krenning EP, van der Gaag R, Koornneef L et al. Prednisone and cyclosporine in the treatment of severe Graves' ophthalmopathy. N Engl J Med 1989; 321: 1353-1359.

6 Werner SC. Classification of the eye changes of Graves' disease. Am J Ophthalmol 1969; 68: 646-648.

7 Gerding MN, van der Zant FM, van Royen EA, Koornneef L, Krenning EP, Wiersinga WM et al. Octreotidescintigraphy is a disease-activity parameter in Graves' ophthalmopathy. Clin Endocrinol 1999; 50: 373-379.

8 Tsai CC, Kau HC, Kao SC, Lin MW, Hsu WM, Liu JH et al. Pulsatile ocular blood flow in patients with Graves' ophthalmopathy. Eye 2005; 19: 159-162.

9 Bartley GB, Gorman CA. Diagnostic Criteria for Graves' Ophthalmopathy. Am J Ophthalmol 1995; 119: 792-795.

10 Silver DM, Farrell RA, Langham ME, O'Brien V, Schilder P. Estimation of pulsatile ocular blood flow from intraocular pressure. Acta Ophthalmol 1989; 191(Suppl): 25-29.

11 Butt Z, O'Brien C. Reproducibility of pulsatile ocular blood flow measurements. J Glaucoma 1995; 4: 214-218.
12 Yang YC, Hulbert MF, Batterbury M, Clearkin LG. Pulsatile ocular blood flow measurements in healthy eyes: reproducibility and reference values. J Glaucoma 1997; 6: 175-179.

13 Chung HS, Harrisa A, Ciulla TA, Kagemann L. Progress in measurement of ocular blood flow and relevance to our understanding of glaucoma and age-related macular degeneration. Prog Retin Eye Res 1999; 18: 669-687.

14 Kerr J, Nelson P, O'Brien C. Pulsatile ocular blood flow in primary open-angle glaucoma and ocular hypertension. Am J Ophthalmol 2003; 136: 1106-1113.

15 Mori F, Konno S, Hikichi T, Yamaguchi Y, Ishiko S, Yoshida A. Pulsatile ocular blood flow study: decreases in exudative age related macular degeneration. Br J Ophthalmol 2001; 85: 531-533.

16 Chen SJ, Cheng CY, Lee AF, Hsu W-M, Liu J-H. Pulsatile ocular blood flow of choroidal neovascularization in asymmetric age-related macular degeneration after transpupillary thermotherapy. Eye 2004; 18: 595-599.

17 Geyer O, Neudorfer M, Snir T, Goldstein M, Rock T, Silver DM et al. Pulsatile ocular blood flow in diabetic retinopathy. Acta Ophthalmol Scand 1999; 77: 522-525.

18 Koornneef L. Eyelid and orbital fascial attachments and their clinical significance. Eye 1988; 2: 130-134.

19 Hudson HL, Levin L, Feldon SE. Graves' exophthalmos unrelated to extraocular muscle enlargement. Superior rectus muscle inflammation may induce venous obstruction. Ophthalmology 1991; 98: 1495-1499.

20 Nakase Y, Osanai T, Yoshikawa K, Inoue Y. Color Doppler imaging of orbital venous flow in dysthyroid optic neuropathy. Jpn J Ophthalmol 1994; 38: 80-86.

21 Somer D, Ozkan SB, Ozdemir H, Atilla S, Soylev MF, Duman S. Color Doppler imaging of superior ophthalmic vein in thyroid-associated eye disease. Jpn J Ophthalmol 2002; 46: 341-345.

22 Li H, Liu YH, Li DH, Zhang Y. Value of measurements of blood flow velocity in central retinal artery in thyroidassociated ophthalmopathy. Zhongguo Yi Xue Ke Xue Yuan Хue Bao 2004; 26: 460-462.

23 Ohtsuka K, Sato A, Kawaguchi S, Hashimoto M, Suzuki Y. Effect of steroid pulse therapy with and without orbital radiotherapy on Graves' ophthalmopathy. Am J Ophthalmol 2003; 135: 285-290. 